\title{
Prevalence and Losses in Quality-adjusted Life Years of Child Health Conditions: A Burden of Disease Analysis
}

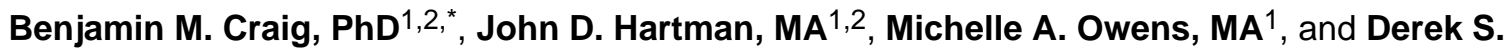 \\ Brown, $\mathrm{PhD}^{3}$ \\ ${ }^{1}$ Moffitt Cancer Center, 12902 Magnolia Drive, MRC-CACONT, Tampa, FL 33612-9416 USA \\ 2University of South Florida, Department of Economics, College of Arts and Sciences, $4202 \mathrm{E}$. \\ Fowler Avenue, CMC206A, Tampa, FL 33612-9416 USA \\ ${ }^{3}$ Washington University in St. Louis, Brown School and Institute for Public Health Campus Box \\ 1196, One Brookings Drive, St. Louis, MO 63130 USA
}

\begin{abstract}
Objectives-To estimate the prevalence and losses in quality-adjusted life years (QALYs) associated with 20 child health conditions.
\end{abstract}

\begin{abstract}
Methods-Using data from the 2009-2010 National Survey of Children with Special Health Care Needs, preference weights were applied to 14 functional difficulties to summarize the quality of life burden of 20 health conditions.
\end{abstract}

\begin{abstract}
Results-Among the 14 functional difficulties, "a little trouble with breathing" had the highest prevalence (37.1\%), but amounted to a loss of just 0.16 QALYs from the perspective of US adults. Though less prevalent, "a lot of behavioral problems" and "chronic pain" were associated with the greatest losses (1.86 and 3.43 QALYs). Among the 20 conditions, allergies and asthma were the most prevalent but were associated with the least burden. Muscular dystrophy and cerebral palsy were among the least prevalent and most burdensome. Furthermore, a scatterplot shows the association between condition prevalence and burden.
\end{abstract}

Conclusions-In child health, condition prevalence is negatively associated with quality of life burden from the perspective of US adults. Both should be considered carefully when evaluating the appropriate role for public health prevention and interventions.

\section{Keywords}

Quality-adjusted life years; child health; children with special health care needs; health preference

\section{INTRODUCTION}

The challenges facing parents and guardians of children with special healthcare needs (CSHCN) on a daily basis are well documented, and include physical, emotional, and

*Address correspondence to: Benjamin Craig, Moffitt Cancer Center, 12902 Magnolia Drive, MRC-CANCONT, Tampa, FL 33612-9416; Phone: (813) 745-6710; Fax: (813) 745-6525; benjamin.craig@ moffitt.org. 
financial impacts.(1-4) The Maternal and Child Health Bureau defines CSHCN as “...those who have or are at increased risk for a chronic physical, developmental, behavioral, or emotional condition and who also require health and related services of a type or amount beyond that required by children generally."(5) To measure the prevalence of US children meeting this definition and the impacts on families, the Centers for Disease Control and Prevention's (CDC) National Center for Health Statistics conducted the National Survey of Children with Special Health Care Needs (NS-CSHCN) 3 times since 2001 under the direction and sponsorship of the federal Maternal and Child Health Bureau. In particular, the 2009-2010 NS-CSHCN conducted more than 40,000 telephone-based interviews asking parents/caregivers a variety of questions about the health and functional status of CSHCN living in their households, including questions on 20 child health conditions and 14 functional difficulties. $(6,7)$

Economic evaluation of services related to CSCHN may help to identify cost-effective approaches for meeting families' needs and for ways to help the greatest number when needs exceed available resources. Good practice for such evaluations(8) requires that both direct financial and indirect, nonfinancial impacts be included, but unfortunately, few studies of the latter exist.(2) The NS-CSHCN is an ideal source for measuring how major conditions affect child health-related quality of life, a central component of indirect costs. However, the NS-CSHCN survey does not summarize functional difficulties related to child health conditions in ways which can be directly used in economic evaluation.(7)

Child health and functional status is particularly difficult to summarize, because the domains cannot be aggregated into a single score (as is required in standard cost-effectiveness analysis)(8) without arbitrarily assigning weights to each domain. For this purpose, the field of "health preference research" has emerged to aid in understanding the value people place on health outcomes, thereby creating preference weights that empirically reflect the perspective of the referent population (e.g., general population, parents). The most common metric involves assessing each outcome in terms of its equivalence in loss of lifespan with no health problems (e.g., full, optimal, best imaginable, or perfect health). The loss of quality-adjusted life years (QALYs) facilitates the summary of health outcomes by aggregating health without reference to treatments, costs, or attributions (e.g., stigma).

In addition, it has been estimated that having a child with a disability can cost society an average of $\$ 30,500$ per year in medical (e.g., health care), non-medical (e.g., reduced caregiver income), and long-term financial costs (e.g., reduced lifetime earnings and employment for the child, increased costs of education).(9) Some caregivers of children with more intensive healthcare needs report spending up to 20 hours per week on direct home care,(10) which reduces the amount of time that can be spent working outside of the home. However, the impacts on parents or guardians of CSHCN vary depending on factors such as type of health condition and functional limitations, financial resources, and caregiver support (e.g., family or friends who may assist with care).(9-13) Undoubtedly, some child health conditions have substantial economic implications in addition to the reductions in child health-related quality of life. This study explores two main research questions concerning the prevalence and losses in QALYs of child health conditions. First, what is the burden of functional difficulties among CSHCN in terms which can be used for future 
economic evaluation-loss in QALYs from the perspective of the general population? Second, what is the relationship between condition prevalence and burden? The prevalence of conditions must be balanced against such a preference-based summary of their burden (i.e., impact of conditions on child health and functional status). By providing this information to clinicians and policy makers we hope to inform future analyses related to childhood health problems. Coupled with expected cost and expected improvements from intervention, this study will help serve as a baseline measure for burdens associated with specific special health care needs. To answer these questions, we combine survey responses from the NS-CSHCN with preference weights from another national survey.

\section{METHODS}

\section{Concept of QALYs}

In non-pediatric applications, a QALY is an idealized year of life with no health problems and is a common preference-based metric used in cost-utility analyses (CUAs) and other forms of comparative effectiveness research (CER) used to inform the allocation of public health resources and health policies.(14-16). While numerous studies have examined QALY values using indirect preferences, the US Panel on Cost-Effectiveness in Health and Medicine prefers a choice-based valuation technique with preferences taken from a nationally representative sample.(17) This method is commonly used to value adult preferences, yet only a handful of studies have examined the value adults place on child health using direct preferences (18-22) and few of these have examined outcomes captured by child-specific health questionnaires or summarized the preferences on a QALY scale. A 2012 valuation study of child health found that US adults would be willing to sacrifice years of adult life to prevent a child from experiencing poor health for a year.(23) As a preferencebased measure, the value of child health outcomes is described relative to QALY losses after childhood. In this paper, we apply preference weights from the perspective of US adults to the child health data gathered in the 2009-2010 NS-CSHCN and examine the relationship between condition prevalence and burden.

\section{9-2010 National Survey of Children with Special Health Care Needs (NS-CSHCN)}

The 2009-2010 NS-CSHCN took approximately 33 minutes to complete (24) and consisted of an introduction, screener, and 3 or 12 additional sections, depending on whether or not a child in the household was identified as having special needs.(25) NS-CSHCN was fielded by telephone in English, Spanish, Mandarin, Cantonese, Vietnamese, and Korean. In total, data were collected from 196,159 households. All children living in each household were screened for special healthcare needs. From these households a total of 372,698 children (ages 0-17) were screened. If no CSHCN were identified, parents were asked a set of demographic questions before the phone call was terminated. If more than one CSHCN lived in a single household, one child was randomly selected to be the participant in the detailed interview. From the 322,145 children, age 3 to 17, that were screened, the Maternal and Child Health Bureau selected 38,034 CSHCN for a detailed interview that included questions on 20 specific conditions and 14 functional difficulties. This analysis excludes children, age 0 to 2, because some questions were not asked due to age inappropriateness (e.g., making friends). All child functional difficulties and conditions were reported by an 
adult and most respondents $(36,507 ; 96 \%)$ completed all 34 questions (Table 1). The 20092010 NS-CSHCN had no differences in completion rate by demographic characteristics, but small differences in poverty level $(<1 \%)$ were observed.

\section{Measurement of burden}

Using the 2009-2010 NS-CSHCN data, we created 20 subsamples representing the 20 childhood health conditions. In each subsample, children had up to 14 functional difficulties. Following standard health economic procedures (26-28), we summarized condition burden by applying preference weights to these functional difficulties and aggregating them. Taken from a previous study,(23) the preference weights for functional difficulties were estimated on a QALY scale based on paired comparison responses, similar to a time trade-off task.(29, 30) An example of a paired comparison question would ask, "which do you prefer: a year with a little difficulty with anxiety followed by 7 years with no health problems or a year with a lot of difficulty with anxiety followed by 10 years with no health problems." Questions such as these are often used to elicit preference weights in the current literature. $(23,31,32)$ As shown in Table 2, preference weights for the 14 functional difficulties measured on NS-CSHCN ranged from 0.059 for a little difficulty seeing to 3.43 for a lot of chronic pain. The latter implies that US respondents were indifferent between a loss of 3.43 QALYs as an adult and a year of suffering for a 10-year-old child (i.e., 50\% prefer the loss of 3.43 QALYs and 50\% prefer the child health outcome).

Prevalence of a child health condition was defined as the proportion of the population with a certain condition at a particular time The average QALY loss is estimated overall (Table 2) and for each of the 20 conditions (Figure 1). Because the preference weights are an expression of US adult values, "burden" in this paper is the amount of adult QALY losses that US adults are willing to sacrifice to prevent a child from experiencing the condition.

\section{Statistical Analysis}

All analyses in this paper are descriptive. To estimate the prevalence of the 20 conditions and 14 functional difficulties, we computed weighted tallies of these items, employing the NS-CSHCN frequency weights, based on the inverse probability of the selection of the phone number that was called, with small adjustments for issues such as multiple cell phone lines, incomplete age-eligibility, and incomplete screener.

\section{RESULTS}

\section{Prevalence and Loss in QALYs of 14 Functional Difficulties among CSHCN}

Table 2 shows the unweighted and weighted prevalence of functional difficulties as well as their value on a QALY scale. A little trouble with breathing had the highest prevalence (37.1\%), followed by a little trouble with anxiety (28.9\%) and learning (27.9\%). A lot of trouble with blood circulation had the lowest prevalence $(0.9 \%)$ followed by a lot of trouble with hearing $(1.5 \%)$. The supplemental appendix document displays the weighted prevalence counts by condition. These results show that CSHCN often suffer from multiple difficulties relating to a variety of different activities in their daily life. The range of difficulties varies highly with each diagnosed condition. As expected, children diagnosed 
from asthma often suffer from breathing problems. However, these children rarely have to deal with coordination issues that plague those diagnosed with cerebral palsy and muscular dystrophy.

Among CSHCN, the average loss was 2.03 QALYs. This suggests that society would be willing to sacrifice 2 QALYs in adulthood to prevent a child from having a year of functional difficulties similar to a child with special health care needs. Of this loss of 2 QALYs, 30\% was due to the prevalence of mild difficulties (i.e., "a little trouble"; 0.62 QALYs) and 70\% is due to severe difficulties (i.e., "a lot of trouble"; 1.41 QALYs). The QALY burden reflects a combination of the prevalence of a difficulty as well as its preference weight. For example, "a lot of trouble" with seeing has the preference weight of 0.359 , but a relatively low prevalence of $4.2 \%$, leading to the average QALY loss of .015 $(0.359 \times 0.042=0.015)$. The most burdensome functional difficulty (i.e., prevalence $\times$ preference weight) was behavior ("a little trouble" 0.14 and "a lot" 0.30 QALYs) followed by chronic pain ( 0.13 and 0.25$)$, anxiety ( 0.08 and 0.25 ) and learning $(0.05$ and 0.23$)$. The least burdensome difficulty (i.e., prevalence $\times$ preference weight) is hearing $(0.005$ and 0.006 ) followed by blood circulation (0.006 and 0.005), seeing (0.007 and 0.015) and use of hands (0.007 and 0.015).

\section{Prevalence and Loss in QALYs of 20 Child Health Conditions}

Figure 1 shows the weighted prevalence and loss in QALYs associated with the 20 child health conditions. The pattern illustrates a negative correlation of .53 between prevalence and loss in QALYs, where the more prevalent conditions have smaller losses, and the least prevalent conditions are associated with greater losses. For example, allergies and asthma are the most prevalent conditions and among the smallest losses; muscular dystrophy is the least prevalent and among the greatest losses. This is consistent with data from the CDC's 2012 National Health Interview Survey (NHIS), which found asthma, allergies, and attention deficit hyperactivity disorder (ADHD) to be among the more prevalent, and muscular dystrophy among the least prevalent, conditions found in US children in the general population. (33)

Although a child with special health care needs has an average of loss of 2.03 QALYs, only $38.4 \%$ of children have special health care needs. Among all children, these 20 health conditions impose a combined burden of 0.78 QALY $(0.384 \times 2.03$ QALYs $)$ from the perspective of US adults. In other words, preventing these 20 conditions for 1 year among all children in the US is estimated to be equivalent to the quality of life gain from improving adult health by 0.78 QALYs. However, the benefits (0.78) are not distributed equally across 20 conditions. Preventing the top 5 most prevalent conditions make up over half of the benefit (0.44): allergies (0.11), ADHD (0.09), delay (0.08), asthma (0.08), and anxiety (0.08). Preventing the remaining 15 conditions is equal to improving adult health by 0.34 QALYs (0.78-0.44).

\section{CONCLUSIONS}

This is the first study to examine the overall nonfinancial population burden of child health conditions on a QALY scale, a metric which is widely used in economic evaluation. We 
remind readers that this burden of disease analysis captures only the nonfinancial burden on the child; it does not account for economic costs (e.g., medical care, lost productivity), or burden on parents/caregivers (e.g., stress, quality of life), or potentially reduced child life expectancy. However, the results of this study provide insight into the perspective of the general population regarding child health conditions and the association between prevalence and burden.

A major innovation of our results is that we have combined preference weights on functional difficulties (23) with national prevalence data captured in the NSCSHCN, leading to aggregate population measures. Previous studies have looked at either the valuation of child health outcomes with no measurement of their population prevalence, such as vignettes of a single condition or health problem. $(20,21,34)$ The results in this paper can therefore be used enhance existing data to make more informed decisions about improving population health through prevention or treatment. Researchers will be able to use these results to compare cost per QALY saved between different interventions to help reduce the burden of CSHCN. The supplemental appendix may prove to be especially helpful for individuals looking to prevent specific problems for one condition. For instance, difficulties with chronic pain currently account for nearly a third of the QALY loss for children with diabetes. A new treatment that would ease the burden for those with chronic pain from a lot to a little would save more than .37 QALYs (17\% of the total loss) per child with a diagnosis of diabetes. A cost-effective analysis could then be performed once researchers determine the costs and potential problems that could be prevented or diminished by certain treatments.

The interpretation of the results must account for 2 different adult perspectives: adults with a CSHCN in the household reporting on the child's health and adults within the general population expressing their preferences on child functional difficulties. On the former, we believe that adults are likely to be able to describe the 14 functional difficulties and the 20 conditions well because the NCSHCN is designed specifically for such measurement. Indeed, adults responsible for their children's care likely to be the most knowledgeable respondents and can probably provide superior data than the child or another adult (e.g., family physician) could in many cases.

Whether or not adults' preferences over child health outcomes should be used in economic models and medical decision-making is unresolved in the literature. $(17,35)$ At least three perspectives are potentially relevant: the child, the parent, and society at large. The child and parent are directly affected by the health problems and arguably have unique insights into living with functional difficulties that should be favored. However, children generally do not make their own health care decisions, do not control the resources associated with these decisions, and may also lack the cognitive capacity to respond properly to valuation and measurement studies. It is difficult to survey children because developmental stage, cognitive ability, and inexperience make it problematic for them to complete preference elicitations tasks.(21) Thus, most of the literature favors an adult perspective, even if preferences may differ. For example, a study by Gerald et al showed that parents' and children's scores on the Pediatric Asthma Health Outcome Measure (PAHOM) differed significantly, and that parent scores were more closely aligned with physician ratings of how well the asthma was controlled.(36) 
At the present time, we are not aware of any study of child preferences or valuations over these outcomes, so we cannot compare how they may differ in this application. Some literature has compared the preferences of parents to all adults in society. Adults in the general population may place a higher value on behavior and learning problems, because of the potential gains for the community. However, it is possible that the values of the general population are similar to those of parents. In one study,(18) parents were asked to value 3 of 29 health states common in pediatric research, provided that their child did not have these conditions. The results of that study were similar to those of this study in that severe cerebral palsy and severe mental retardation had the lowest utility values (i.e., were considered most burdensome). Another study found that the global burden of autism spectrum disorders was is the leading cause of disability when prevalence was considered.(37)

The health preferences of children, parents, or survivors of childhood diseases may differ from those of the US adult population as a whole. This would be important if, for instance, the results were to be used to help parents of CSHCN decide how to focus limited resources in addressing child functional difficulties in order to achieve the maximum possible quality of life benefit; or, if two alternative intervention programs were available, both with outcomes data (measured by the 14 difficulties), and one wanted to see which was more effective in meeting the needs of parents, children, or survivors. Although we hope to examine these preferences in future work, US adults are the primary stakeholders (taxpayers) in resource allocation decisions and economic evaluations at the broadest possible level, such as local, state, or national public health programs. Alternatively, parents may be used as a proxy to assess familial preferences; however, this complicates the interpretation, particularly when outcomes relate to domestic violence or when the household contains multiple children.

Our study has several important limitations. The issues of perspective and reporting by parents were discussed above, although these are not necessarily negatives. Second, children may have multiple health conditions (38-40) and children with a health condition often have multiple functional limitations. $(41,42)$ For example, chronic physical conditions have been shown to be a significant risk factor for behavior problems, (43) and ADHD is often associated with anxiety/depression and problems with usual activities.(21) This study did not separate the burden of children with multiple conditions because of the high number of possible interactions. Related to this, our study-like virtually HRQOL applications in health economics-necessarily relies on the original valuation study.(23) Those results not only directly affect our findings here, they limit us in terms of multiple functional difficulties, because they do not separately examine the interaction between difficulties (e.g., does the value of learning problems decrease if the child is in severe pain?). Finally, we did not conduct a multivariate analysis or other statistical testing to try to isolate the relationship between the 20 health conditions and the responses to the 14 functional difficulties. Given the extremely limited national prevalence and burden literature on CSHCN, we feel that a descriptive analysis is most appropriate at this stage, although additional analysis may be considered in future research. Regardless of these limitations, the study demonstrates the potential public health gains that may result from helping CSHCN. 


\section{Supplementary Material}

Refer to Web version on PubMed Central for supplementary material.

\section{Acknowledgments}

Funding: Funding support for this research was provided by a grant from the National Institutes of Health, Department of Health and Human Services, through the National Cancer Institute (1R01CA160104) and Dr. Craig's support account at Moffitt Cancer Center.

The authors thank Catherine Blackburn and Carol Templeton for their contributions to references and editing of this paper, respectively.

\section{References}

1. Bumbalo J, Ustinich L, Ramcharran D, et al. Economic impact on families caring for children with special health care needs in New Hampshire: The effect of socioeconomic and health-related factors. Matern Child Health J. 2005; 9(2):S3-S11.10.1007/s10995-005-4350-3 [PubMed: 15973476]

2. DeRigne L. The Employment and Financial Effects on Families Raising Children With Special Health Care Needs: An Examination of the Evidence. J Pediatr Health Care. 2012; 26(4):28390.10.1016/j.pedhc.2010.12.006 [PubMed: 22726713]

3. McIntyre LL, Blacher J, Baker BL. Behaviour/mental health problems in young adults with intellectual disability: the impact on families. J Intellect Disabil Res. 2002; 46:239-49.10.1046/j. 1365-2788.2002.00371.x [PubMed: 11896809]

4. Quach J, Oberklaid F, Gold L, et al. Primary health-care costs associated with special health care needs up to age 7 years: Australian population-based study. J Paediatr Child Health. 2014; 50(10): 768-74.10.1111/jpc.12649 [PubMed: 24923806]

5. McPherson M, Arango P, Fox H, et al. A new definition of children with special health care needs. Pediatrics. 1998; 102(1):137-40.10.1542/peds.102.1.137 [PubMed: 9714637]

6. Centers for Disease Control and Prevention (CDC). State and Local Area Integrated Telephone Survey-National Survey of Children with Special Health Care Needs. CDC/National Center for Health Statistics; http://www.cdc.gov/nchs/slaits/cshcn.htmhttp://www.cdc.gov/nchs/slaits/ cshcn.htm [Access: 9/5/2014Accessed 9/5/2014]

7. Child and Adolescent Health Measurement Initiative. 2009/10 NS-CSHCN: Health Conditions and Functional Difficulties. Data Resource Center, supported by Cooperative Agreement 1-U59MC06980-01 from the US Department of Health and Human Services, Health Resources and Services Administration (HRSA), Maternal and Child Health Bureau (MCHB). 2012. Available at www.childhealthdata.org, Revised 01/27/2012

8. Gold, MR.; Siegel, JE.; Russell, LB.; Weinstein, MC., editors. Cost-Effectivenss in Health and Medicine. New York: Oxford University Press; 1996.

9. Stabile M, Allin S. The Economic Costs of Childhood Disability. Future Child. 2012; 22(1):65-96. [PubMed: 22550686]

10. Kuo DZ, Cohen E, Agrawal R, et al. A National Profile of Caregiver Challenges Among More Medically Complex Children With Special Health Care Needs. Arch Pediatr Adolesc Med. 2011; 165(11):1020-6. [PubMed: 22065182]

11. Smith AL, Romski M, Sevcik RA, et al. Parent Stress and Perceptions of Language Development: Comparing Down Syndrome and Other Developmental Disabilities. Fam Relat. 2014; 63(1):7184.10.1111/fare.12048 [PubMed: 24753637]

12. Szilagyi PG. Health Insurance and Children with Disabilities. Future Child. 2012; 22(1):123-48. [PubMed: 22550688]

13. Viana MC, Gruber MJ, Shahly V, et al. Family burden related to mental and physical disorders in the world: results from the WHO World Mental Health (WMH) surveys. Rev Bras Psiquiatr. 2013; 35(2):115-25. [PubMed: 23904015] 
14. Drummond M, Brixner D, Gold M, et al. Toward a Consensus on the QALY. Value Health. 2009; 12:S31-S5.10.1111/j.1524-4733.2009.00522.x [PubMed: 19250129]

15. Fryback DG, Lawrence WF Jr. Dollars may not buy as many QALYs as we think: a problem with defining quality-of-life adjustments. Med Decis Making. 1997; 17(3):276-84. [PubMed: 9219187]

16. World Health Organization (WHO). Essential Medicines and Health Products Information Portal. Introduction to Drug Utilization Research. 2003

17. Griebsch I, Coast J, Brown J. Quality-adjusted life-years lack quality in pediatric care: A critical review of published cost-utility studies in child health. Pediatrics. 2005; 115(5):E600E14.10.1542/peds.2004-2127 [PubMed: 15867026]

18. Carroll AE, Downs SM. Improving Decision Analyses: Parent Preferences (Utility Values) for Pediatric Health Outcomes. J Pediatr. 2009; 155(1):21-5.10.1016/j.jpeds.2009.01.040 [PubMed: 19394030]

19. Friedman JY, Reed SD, Weinfurt KP, et al. Parents' reported preference scores for childhood atopic dermatitis disease states. BMC pediatrics. 2004; 4(1):21.10.1186/1471-2431-4-21 [PubMed: 15491500]

20. Hauber AB, Itzler R, Johnson FR, et al. Healthy-days time equivalents for outcomes of acute rotavirus infections. Vaccine. 2011; 29(45):8086-93.10.1016/j.vaccine.2011.08.041 [PubMed: 21864612]

21. Secnik K, Matza LS, Cottrell S, et al. Health state utilities for childhood attention-deficit/ hyperactivity-disorder based on parent preferences in the United Kingdom. Med Decis Mak. 2005; 25(1):56-70.10.1177/0272989x04273140

22. Ungar, W. Economic evaluation in child health. Oxford: Oxford University Press; 2010.

23. Craig BM, Brown DS, Reeve BB. The Value Adults Place on Child Health and Functional Status. Value Health. 2015; 18(4):449-56.10.1016/j.jval.2015.02.012 [PubMed: 26091599]

24. Data Resource Center for Child \& Adolescent Health. [Accessed date: 9/26/2014] National Surey of Children with Speacial Health Care Needs (2009/10 NS-CSHCN): FAST FACTS about the SURVEY. http://childhealthdata.org/docs/default-document-library/ns-cshcn-fastfacts-2009.pdfhttp://childhealthdata.org/docs/default-document-library/ns-cshcn-fastfacts-2009.pdf

25. National Center for Health Statistics. Centers for Disease Control and Prevention. [Access: 9/26/2014Accessed September 26, 2014] 2009-2010 National Survey of Children with Special Health Care Needs. http://www.cdc.gov/nchs/data/slaits/ NS_CSHCN_Questionnaire_09_10.pdfhttp://www.cdc.gov/nchs/data/slaits/ NS_CSHCN_Questionnaire_09_10.pdf

26. Mulhern B, Bansback N, Brazier J, et al. Preparatory study for the revaluation of the EQ-5D tariff: methodology report. Health Technol Assess. 2014; 18(12):1-191.10.3310/hta18120 [PubMed: 24524660]

27. Petrou S, Kandala NB, Robinson A, et al. A Person Trade-Off Study to Estimate Age-Related Weights for Health Gains in Economic Evaluation. PharmacoEconomics. 2013; 31(10):893907.10.1007/s40273-013-0085-y [PubMed: 23990389]

28. Prieto L, Sacristan JA. Problems and solutions in calculating quality-adjusted life years (QALYs). Health and quality of life outcomes. 2003; 110.1186/1477-7525-1-80

29. Lenert L, Kaplan RM. Validity and interpretation of preference-based measures of health-related quality of life. Med Care. 2000; 38(9):138-50.

30. Torrance GW. Utility approach to measuring health-related quality of life. J Chronic Dis. 1987; 40(6):593-603. [PubMed: 3298297]

31. Craig BM, Brown DS, Reeve B. Valuation of Child Behavioral Problems from the Perspective of US Adults. Med Decis Mak. In Press.

32. Craig BM, Reeve BB, Brown PM, et al. US Valuation of Health Outcomes Measured Using the PROMIS-29. Value Health. 2014; 17(8):846-53.10.1016/j.jval.2014.09.005 [PubMed: 25498780]

33. Bloom B, Jones LI, Freeman G. Summary health statistics for US children: National Health Interview Survey, 2012. 2013 
34. Ladapo JA, Neumann PJ, Keren R, et al. Valuing children's health: a comparison of cost-utility analyses for adult and paediatric health interventions in the US. PharmacoEconomics. 2007; 25(10):817-28. [PubMed: 17887804]

35. Ungar WJ. Challenges in Health State Valuation in Paediatric Economic Evaluation Are QALYs Contraindicated? PharmacoEconomics. 2011; 29(8):641-52. [PubMed: 21604821]

36. Gerald JK, McClure LA, Harrington KF, et al. Measurement Characteristics of the Pediatric Asthma Health Outcome Measure. J Asthma. 2012; 49(3):260-6.10.3109/02770903.2012.656863 [PubMed: 22335297]

37. Baxter AJ, Brugha TS, Erskine HE, et al. The epidemiology and global burden of autism spectrum disorders. Psychol Med. 2014:1-13.10.1017/s003329171400172x

38. Arbelle JE, Chodick G, Goldstein A, et al. Multiple chronic disorders - health care system's modern challenge in the Maccabi Health Care System. Isr J Health Policy Res. 2014; 3:29.10.1186/2045-4015-3-29 [PubMed: 25206971]

39. Bitsko RH, Visser SN, Schieve LA, et al. Unmet health care needs among CSHCN with neurologic conditions. Pediatrics. 2009; 124(Suppl 4):S343-51.10.1542/peds.2009-1255D [PubMed: 19948598]

40. Zhong W, Finnie DM, Shah ND, et al. Effect of Multiple Chronic Diseases on Health Care Expenditures in Childhood. J Prim Care Community Health. 201410.1177/2150131914540916

41. Blackman JA, Gurka MJ, Gurka KK, et al. Emotional, developmental and behavioural comorbidities of children with chronic health conditions. J Paediatr Child Health. 2011; 47(10):7427.10.1111/j.1440-1754.2011.02044.x [PubMed: 21449905]

42. Steele R, Siden H, Cadell S, et al. Charting the territory: symptoms and functional assessment in children with progressive, non-curable conditions. Arch Dis Child. 2014; 99(8):754-62.10.1136/ archdischild-2013-305246 [PubMed: 24833792]

43. Gortmaker SL, Walker DK, Weitzman M, et al. Chronic conditions, socioeconomic risks, and behavioral problems in children and adolescents. Pediatrics. 1990; 85(3):267-76. [PubMed: 2304779] 


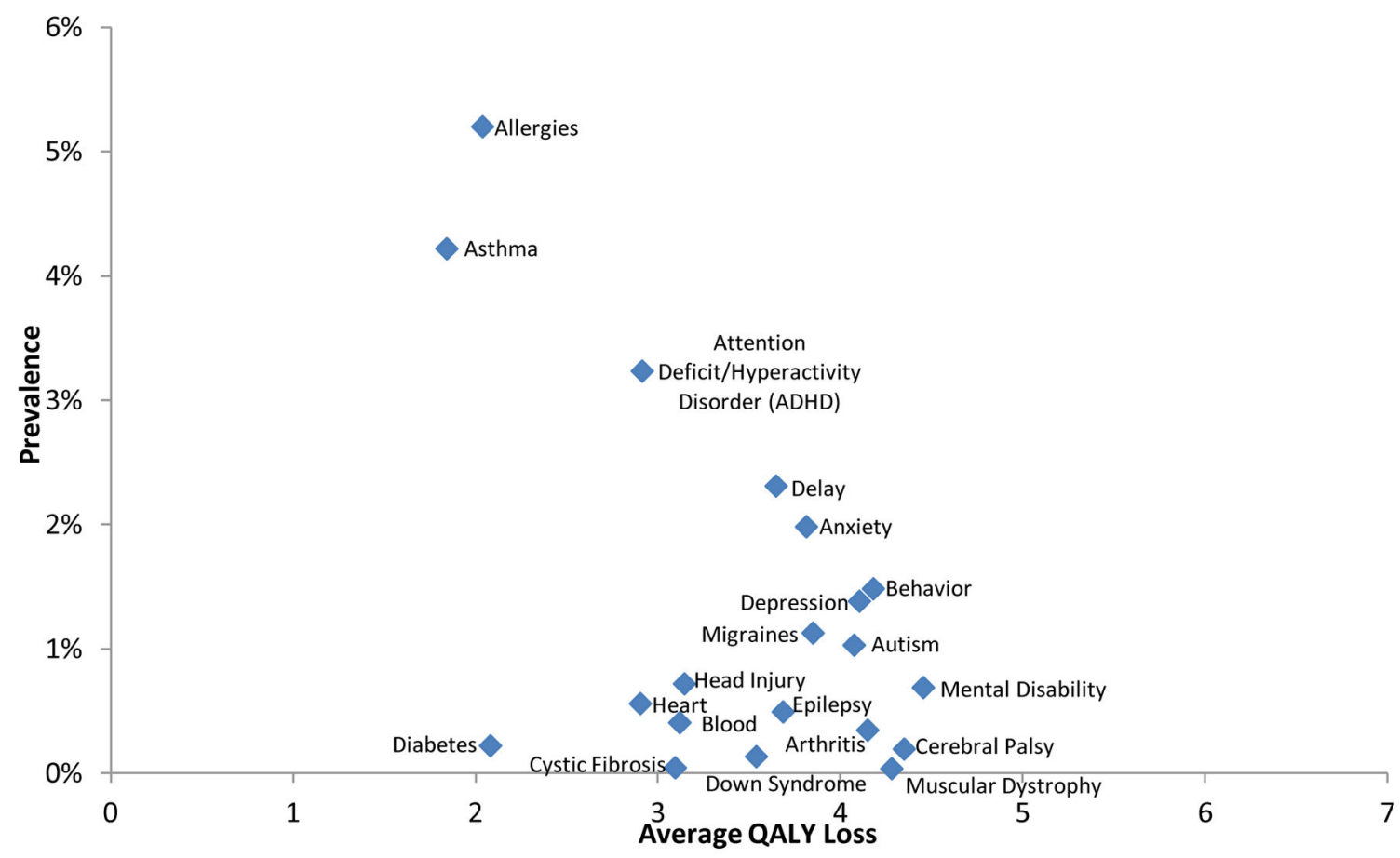

Figure 1.

Child Health Conditions: Prevalence and Average Loss in Quality-adjusted Life Years (QALYs)*

* "For each condition, please tell me if a doctor or other health care provider ever told you that [child's name] had the condition, even if [he/she] does not have the condition now." 


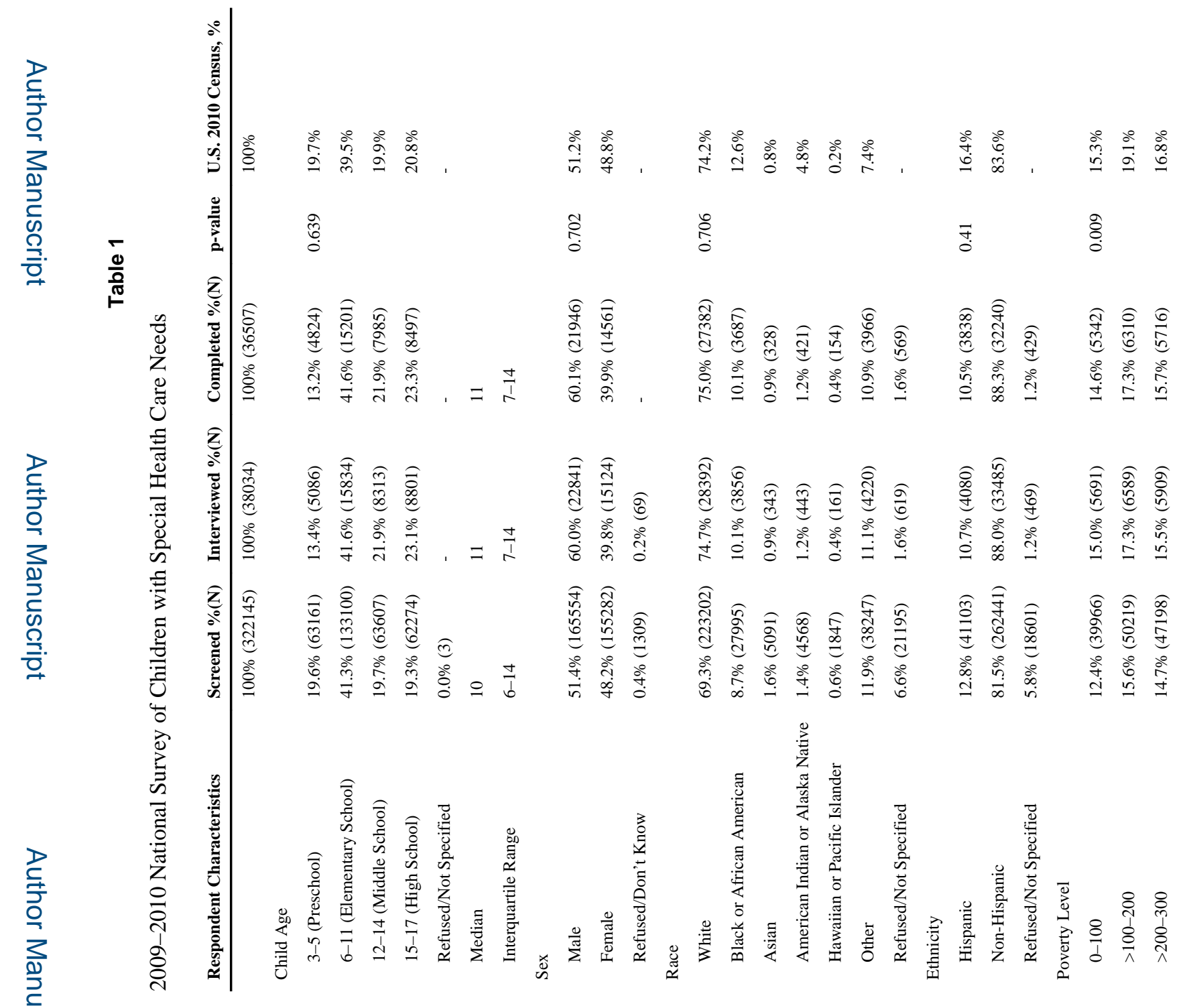

Matern Child Health J. Author manuscript; available in PMC 2017 April 01. 


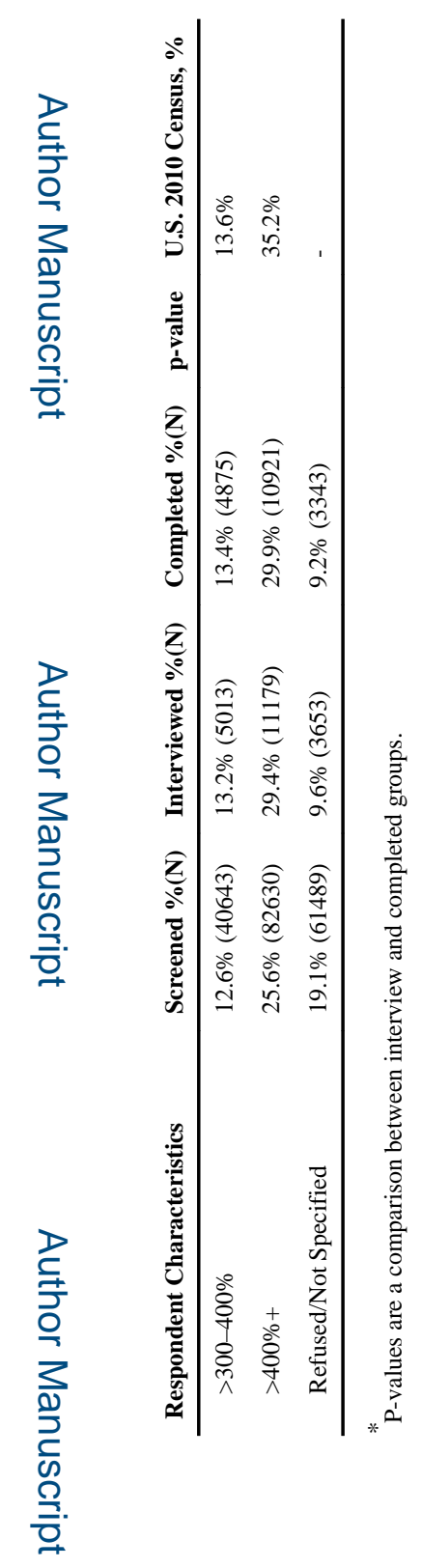




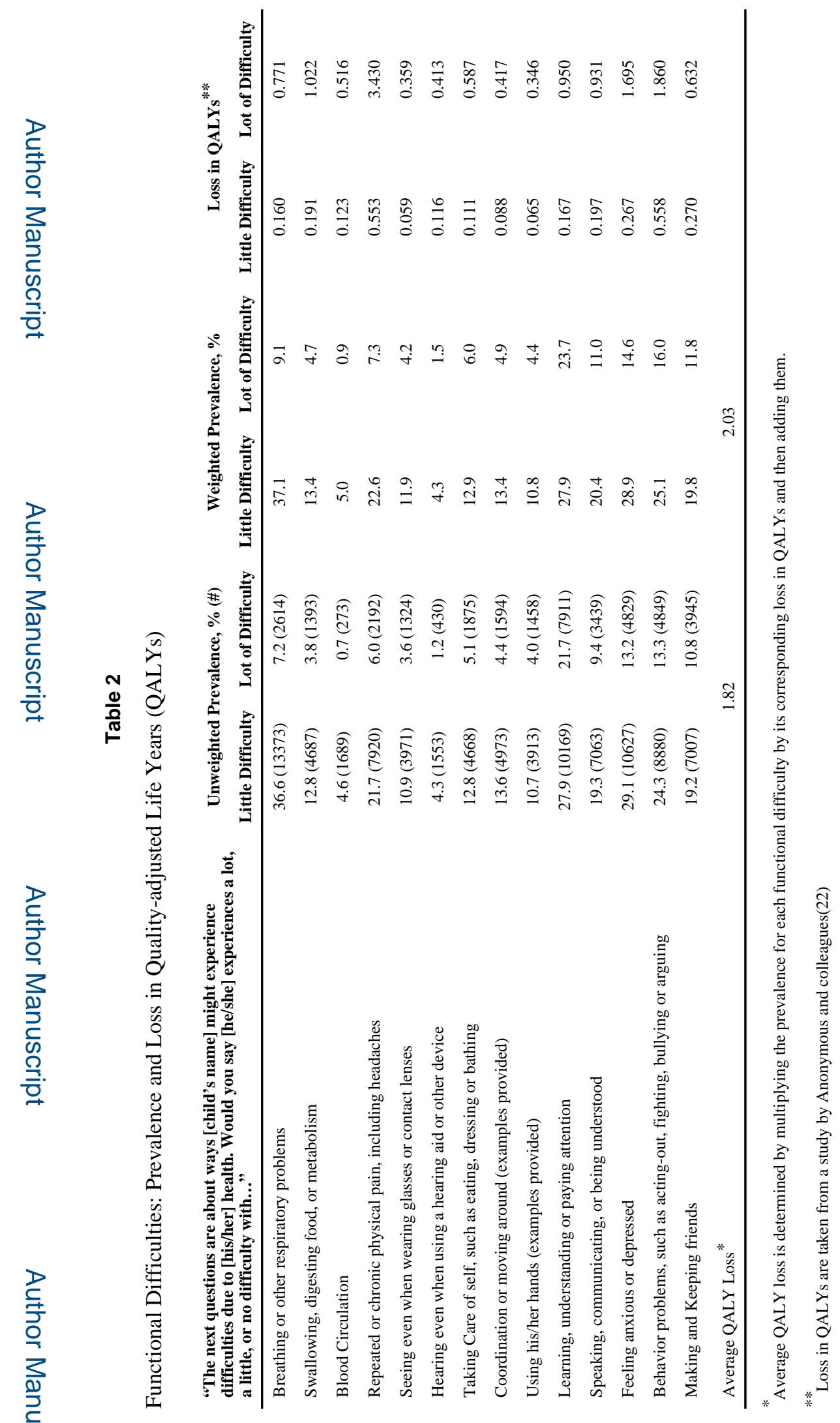

Matern Child Health J. Author manuscript; available in PMC 2017 April 01. 


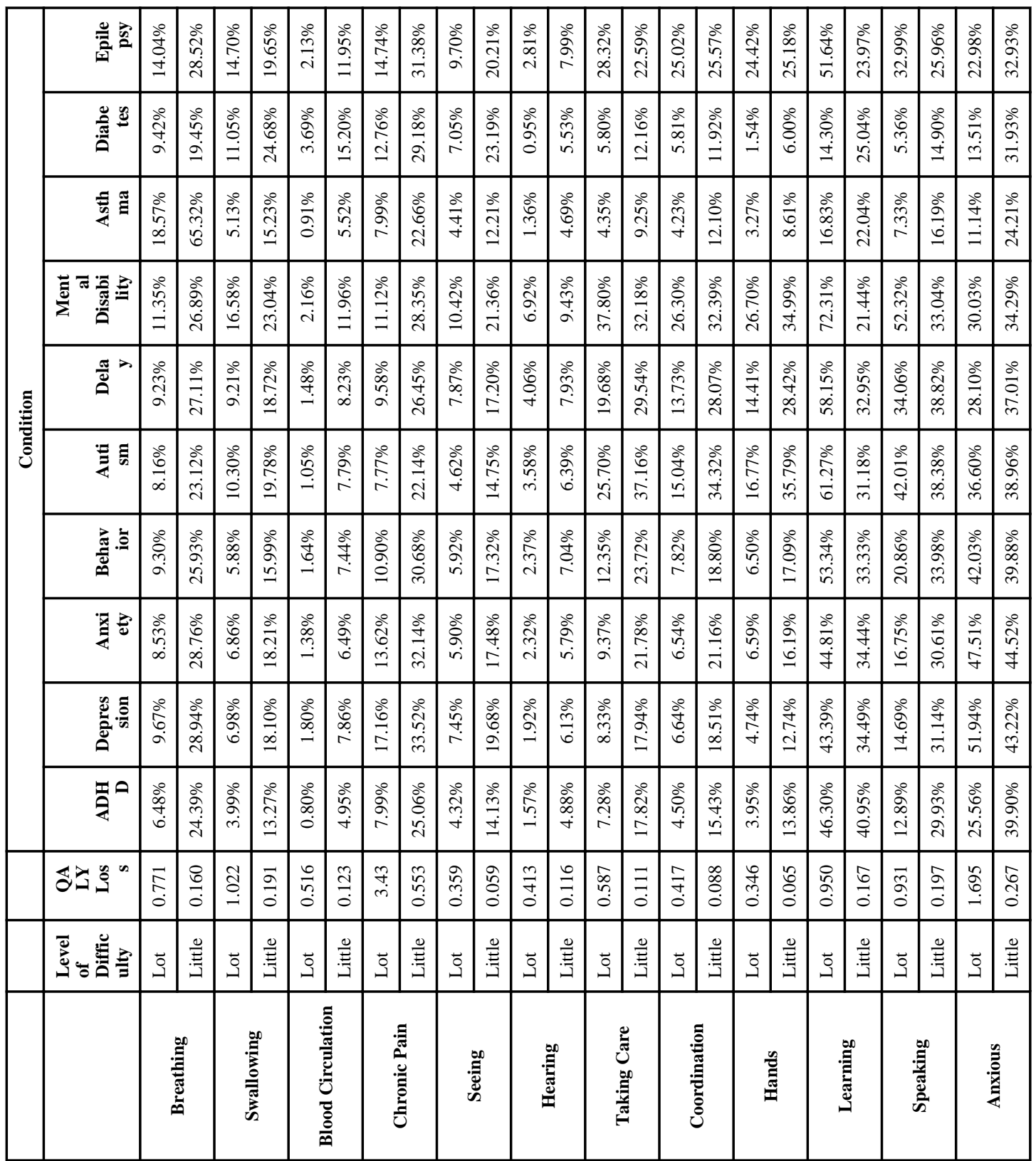

Matern Child Health J. Author manuscript; available in PMC 2017 April 01. 


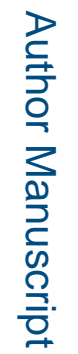

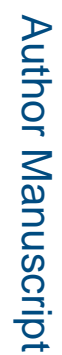

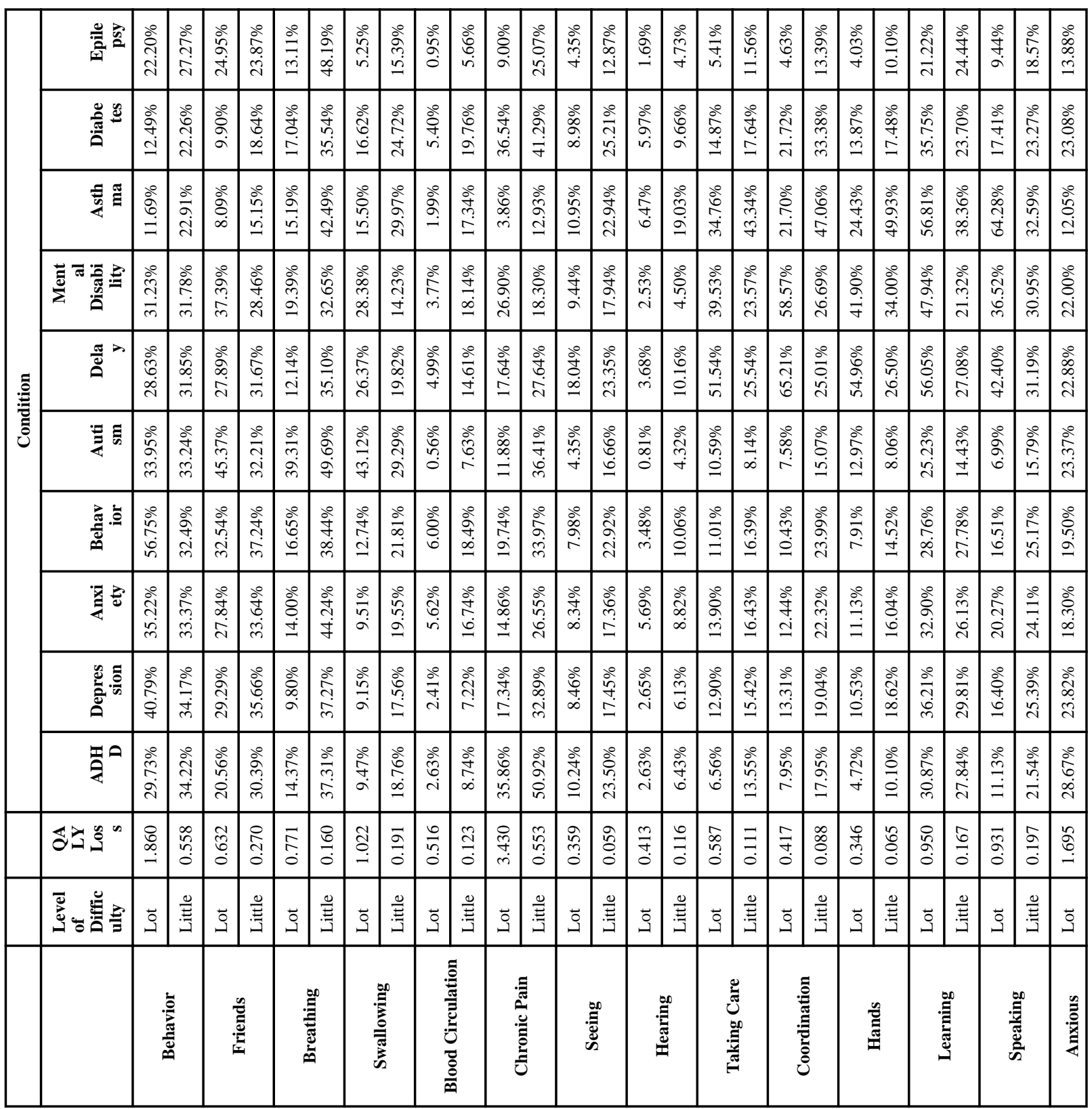

Matern Child Health J. Author manuscript; available in PMC 2017 April 01. 


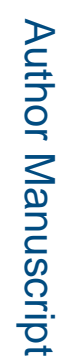

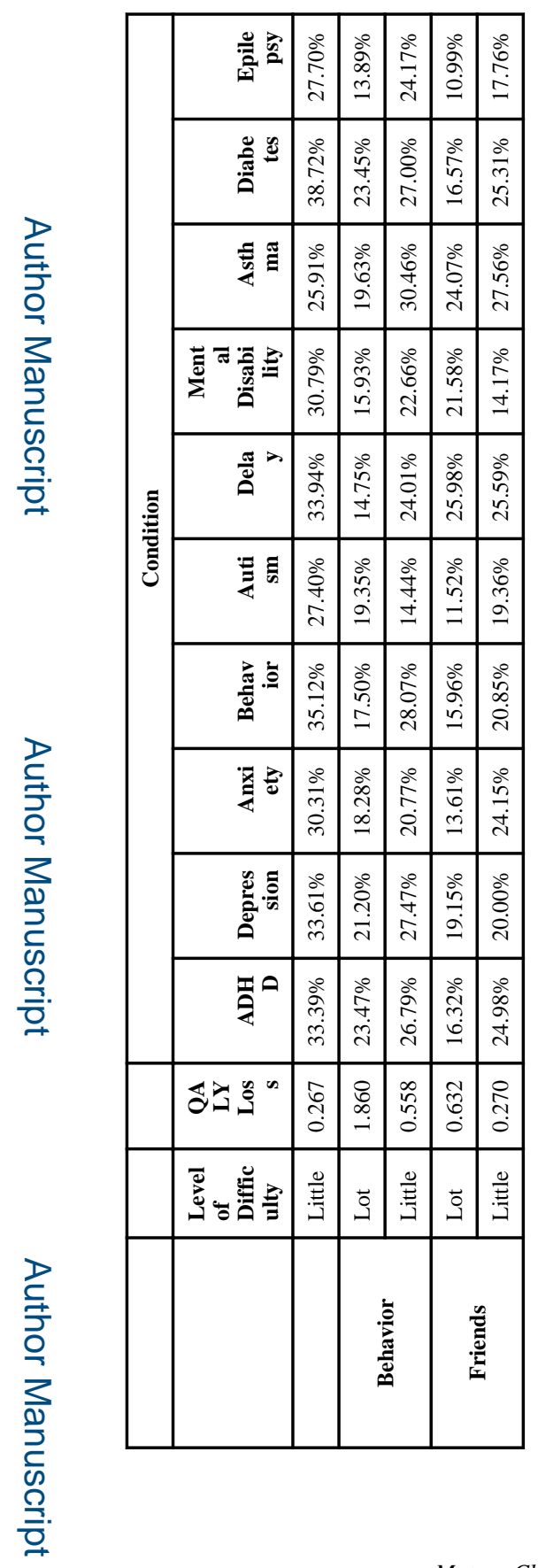

Matern Child Health J. Author manuscript; available in PMC 2017 April 01. 